\title{
Diálisis Peritoneal Intermitente en Pediatría
}

\author{
Dr. Carlos Saieh A. ${ }^{1-4}$; Dr. José Baeza R. ${ }^{2}$; Dr. Jaime Cotdero T. ${ }^{2}$; \\ Dr. Eugenio Rodríguez S. ${ }^{1}$; Dr. Federico Puga C. ${ }^{1-4}$; Dr. Mario Varela G. ${ }^{1}$; \\ E.U. Carmen Hernández F 3 ; E.U. Janet González M. ${ }^{3}$
}

\section{Intermitent Peritoneal Dialysis in Childnen}

Eight children with end-stage renal falure were treated by intermittent peritoneal dialysis (IPD) from 1983 to 1985 in a Metropolitan hospital at Santiago, Chile. Six females and two males ranging 2 years 1 month to 12 years 10 months of age were included. The Tenckhoff catheter was used and all the patients were mantained on peritoneal dialysis every 3 days, during 24 hours, for periods of 1 to 7 months. Anthropometric, bjochemical and hematological evaluations were performed twice a month. Six out of 8 patients were grafted with kidneys from live related donnors, the other two are waiting a donnor. Peritonitis was detected in a 3 patients, and catheter obstruction ocurred in other two children. It is concluded that IPD is useful in the treatment of uremic children and is valuable in end-stage renal failure waiting for transplantation.

(Key words: Peritoneal dialysis. Renal failure).

La diálisis peritoneal ha tenido una buena aceptación entre los pediatras debido a los resul. tados obtenidos en comparación con la hemodiálisis 1,2 . En muchos países se usa la peritoneo diálisis ambulatoria como un procedimiento que ha salvado innumerables vidas $y$ ha permitido realizar transplantes renales en mejores condiciones. La mayor experiencia existe con el uso de la diálisis peritoneal continua ambulatoria (DPCA) y en menor escala con diálisis peritoneal intermitente (DPI), aunque esta última data de $1962^{3}$. La DPI se realiza habitualmente con sistemas automatizados, que han permitido obtener resultados muy satisfactorios dado lo eficiente del sistema y la poca incidencia de complicaciones ${ }^{4-7}$, aunque con DPI manual se han publicado experiencias simitares $^{8,9}$.

Durante los últimos años se han efectuado varias publicaciones acerca de la peritoneo diálisis en niños con insuficiencia renal crónica terminal, y recientemente Fine y Gruskin resumen esta experiencia desde el punto de vista histórico,

1. Departamento de Nefro-urología, Hospital Luis Calvo Mackenna.

2. Unidad de Tratamiento Intensivo, Hospital Luis Calvo Mackenna.

3. Enfermera Uhidad de Tratamiento Intensivo, Hospital Luis Calyo Mackenna.

4. Departamento de Pediatr ía, Divisiớn Ciencias Médicas Oriente, Facultad de Medicina, Lniversidad de Chile, fisiológico, clínico y terapéutico, tanto de la peritoneo diálisis como de la hemodiálisis, dando incluso un gran énfasis al trasplante renal y a sus aspectos sicosociales ${ }^{1}$.

El objetivo de esta comunicación es mostrar los resultados del programa de peritoneo diálisis crónica intermitente realizado en los pacientes insuficientes renales crónicos terminales antes del trasplante renal en los últimos dos años en el hospital Luis Calvo Mackenna.

\section{MATERIAL Y METODO}

Se evaluaron los resultados obtenidos en 8 pacientes, 6 mujeres, que ingresaron al programa desde septiembre de 1983 a mayo de 1985 en el Hospital Luis Calvo Mackenna, cuyas edades fluctuaron entre 25 y 154 meses, con un promedio de 112 meses.

El diagnóstico de insuficiencia renal terminal fue considecado, cuando la depuración de crea. tinina estaba por debajo de $10 \mathrm{ml} \times \mathrm{min} \times 1,73$ $\mathrm{m}^{2}$ superficie corporal en forma mantenida por un período no inferior a 3 meses con repercusión clínico-metabólica, y respuesta poco satisfactoria al tratamiento médico habitual.

Las características de cada uno de los pacientes en el momento de ingresar al programa de DPI se resumen en la Tabla 1.

La dieta fue absolutamente libre mientras los nifios estuvieron en diálisis en el hospital y sólo 
Tabla 1

Características Clínicas de 8 Pacientes al Inicio de Diálisis Peritoneal Intermitente

\begin{tabular}{ccccccc}
\hline $\begin{array}{c}\text { Paciente } \\
N^{\circ}\end{array}$ & $\begin{array}{c}\text { Edad } \\
\text { Años/meses }\end{array}$ & $\begin{array}{c}\text { Cl. Cr. } \\
\mathrm{ml} / \mathrm{min}^{\prime} \\
1,73 \mathrm{~m}^{2}\end{array}$ & $\begin{array}{c}\text { Peso/Edad } \\
\%\end{array}$ & $\begin{array}{c}\text { Peso/Talla } \\
\%\end{array}$ & $\begin{array}{c}\text { Pr. Art. } \\
\text { mm Hg. }\end{array}$ & $\begin{array}{c}\text { Tiempo en } \\
\text { Diálisis } \\
\text { meses (actual) }\end{array}$ \\
\hline 1 & $2 / 1$ & 8,0 & 78,0 & 98,6 & $100 / 60$ & 4 \\
2 & $10 / 6$ & 6,5 & 77,0 & 104,0 & $120 / 80$ & 1 \\
3 & $9 / 8$ & 5,5 & 89,0 & 100,0 & $130 / 90$ & 3 \\
4 & $9 / 3$ & 7,5 & 79,0 & 101,0 & $120 / 80$ & 7,5 \\
5 & $7 / 6$ & 5,3 & 85,8 & 94,7 & $160 / 130$ & 5 \\
7 & $12 / 10$ & 5,3 & 92,0 & 90,4 & $150 / 100$ & 7,5 \\
8 & $12 / 6$ & 9,7 & 94,0 & 94,0 & $130 / 90$ & 3 \\
\hline
\end{tabular}

fue restringid a en sal en los sujetos hipertensos.

Los pacientes fueron sometidos a mediciones antropométricas cada 15 días (peso, talla, pliegues cutáneos), bioquímicas (creatinina, nitró. geno ureico, electrolitos, gases en sangre, calcemia, fosfemia, colesterolemia, albuminemia) y hematológicas (hematocrito y hemoglobina).

En todos ellos se instaló un catéter de Silastic (Tenchhoff) con adaptador de titanio. El procedimiento fue efectuado bajo anestesia general en pabellón por el equipo de urología de nuestro hospital, de acuerdo a técnicas previamente descritas $17-20$.

Como líquido de diálisis se empleó una solu. ción glucosa y electrolitos de composición semejante al plasma, a $37^{\circ} \mathrm{C}$, manteniéndola en la cavidad peritoneal durante 45 minutos y removiéndola en 15 minutos: esta maniobra se repitió 24 veces en cada procedimiento. La cantidad de liquido que se uso fue variable y de acuerdo a la tolerancia; así; en recién nacidos, los rafigos usados pueden variar desde 20 a $100 \mathrm{ml}$ por kilo, en el lactante y pre-escolar entre $20 \mathrm{y}$ $50 \mathrm{ml} \mathrm{y}$ en el niño mayor se aceptan volúmenes que varían entre 30 y $50 \mathrm{ml}$ por kilo, con un máximo de $2.000 \mathrm{ml}$ por baño $11-15$

\section{RESULTADOS}

Durante el período de estudio se efectuaron 268 diálisis peritoneales de 24 horas cada una, con un mínimo de 10 y un máximo de 60 por paciente, en forma períodica 2 veces por semana.
De los 8 pacientes que ingresaron al programa hasta el momento, 6 han recibido transplantes. Los 2 pacientes restantes, que son los que cum. plen los períodos más prolongados de diálisis, se encuent ran en espera de donante.

El peso, libre de edema, mostró significativos incrementos en 6 de los 7 pacientes dializados por tres meses o más. Un paciente con anorexia pertinaz, trastornos reactivos emocionales y rechazo del procedimiento mantuvo una curva ponderal plana, sin relación con la mejoría de los parámetros bioquímicos. Los incrementos en la talla son difíciles de valorar dado el corto perío. do de estudio en la mayoría (promedio 4 meses) de los casos.

La cantidad de calorías en forma de glucosa absorbida a través del dializado fue estimada en 200 a 400 calorías $x$ día de diálisis, dependiendo del volumen empleado. Las pérdidas de proteinas (albúmina) habitualmente correspondieron a 0,3 ó $0,5 \mathrm{~g} \mathrm{x} 1$ elevándose a valores de hasta $1,75 \mathrm{~g} \mathrm{x}$ 1 en caso de infección peritoneal.

Salvo un paciente que tuvo hipoalbuminemia sostenida, los procedimientos fueron efectivos para mantener a los pacientes sin edema clínico.

Dos pacientes de 4 que ingresaron al programa con hipertensión, requirieron, durante los procedimientos, dosis máximas de hipotensores y asociación de ellos para controlar su presión arterial: Furosemida $3 \mathrm{mg} x \mathrm{~kg} x$ dosis, propranolol 3 a 5 $\mathrm{mg} \times \mathrm{kg} \times$ dia e Hidrazalina $3 \mathrm{mg} \times \mathrm{kg} x$ día. En un caso se usó además Captopril en dosis de $2 \mathrm{mg}$ $\mathrm{x} \mathrm{kg} \times \mathrm{x}$ por dia.

En la Tabla 2 se expresan y comparan las cifras iniciales y luego de tres meses de diálisis de 
Tabla 2

Comparaciones de Algunos Parámetros Bioquímicos y Evolución Ponderat al Ingreso y a los 3 Meses do Diálisis Peritoneal Intermitente en Sietc Pacientes

\begin{tabular}{|c|c|c|c|c|c|c|c|c|c|c|c|c|c|c|}
\hline \multirow[t]{2}{*}{ PACIENTE No } & \multicolumn{2}{|c|}{1} & \multicolumn{2}{|c|}{3} & \multicolumn{2}{|l|}{4} & \multicolumn{2}{|l|}{5} & \multicolumn{2}{|l|}{6} & \multicolumn{2}{|c|}{7} & \multicolumn{2}{|l|}{8} \\
\hline & I & $\mathbf{C}$ & I & $\mathrm{C}$ & $\mathbf{1}$ & C & I & $\mathrm{C}$ & I & $\mathrm{C}$ & 1 & $\mathrm{C}$ & $\mathbf{1}$ & $\mathrm{c}$ \\
\hline \multirow{2}{*}{$\mathrm{N}$, UREICO (mg \%) } & & & & $78,6 \pm$ & $67,1 \pm$ & $60,2 \pm$ & $175,6 \pm$ & $68,2 \pm$ & $68,5 \pm$ & $36,5 \pm$ & $166,2 \pm$ & $68,9 \pm$ & $16,9 \pm$ & \\
\hline & 21,0 & 20.1 & 36,0 & 23,8 & 10,8 & 6,3 & 5,3 & 9,7 & 3,8 & 15,9 & 26,4 & 9.7 & 28,0 & 11,7 \\
\hline \multirow{2}{*}{ CREATININEMIA $(\mathrm{mg} \%)$} & $6,3 \pm$ & $3,0 \pm$ & $11,0 \pm$ & $7,6 \pm$ & $6,8 \pm$ & $5,4 \pm$ & $10,0 \pm$ & $10,1 \pm$ & $13,2 \pm$ & $7,1 \ddagger$ & $7,5 \pm$ & $6,2 \pm$ & $16,2 \pm$ & $8,2 \pm$ \\
\hline & 1,8 & 0,2 & 0,9 & 0,9 & 0,6 & 0,3 & 1,2 & 1,5 & 1,2 & 1,5 & 1,3 & 0,3 & 1,8 & 0,6 \\
\hline \multirow{2}{*}{ CALCFMIA (mg\%) } & $5,7 \pm$ & $8,9 \pm$ & $5,8 \pm$ & $7.6 \pm$ & $5,6 \pm$ & $8,5 \pm$ & $7,2 \pm$ & $8,4 \pm$ & $5,2 \pm$ & $8,6 \pm$ & $9,1 \pm$ & $8,5 \pm$ & $4,2 \pm$ & $9,7 \pm$ \\
\hline & 0,9 & 0,4 & 1,1 & 0,3 & 0,6 & 0,7 & 0,6 & 1,0 & 0,8 & 0,2 & 0,7 & 1,5 & 0,6 & 0,8 \\
\hline \multirow{2}{*}{ FOSFEMIA (mg \%) } & $8,7 \pm$ & $4,4 \pm$ & $6.5 \pm$ & $5,4 \pm$ & $5,9 \pm$ & $5,9 \pm$ & $11,3 \pm$ & $6,3 \pm$ & $10,0 \pm$ & $4,5 \pm$ & $3,9 \pm$ & $6,3 \pm$ & $10,0 \pm$ & $6,3 \pm$ \\
\hline & 1.4 & 0,5 & 3,0 & 0,8 & 1,2 & 0,8 & 1,3 & 0,7 & 1,3 & 0,3 & 0,9 & 0,7 & 0.6 & 1,1 \\
\hline \multirow{2}{*}{ ALBUMINEMIA $(\mathrm{gr} \%)$} & $41,2 \pm$ & $35,7 \pm$ & $33,8 \pm$ & $33,5 \pm$ & $45,5 \pm$ & $40,8 \pm$ & $29,6 \pm$ & $27,5 \pm$ & $30,8 \pm$ & $28,2 \pm$ & $31,3 \pm$ & $33,0 \pm$ & $27,7 \pm$ & $35,1 \pm$ \\
\hline & 1,4 & 0,3 & 1,2 & 0.7 & 1,8 & 1,3 & 0,8 & 1,4 & 1,6 & 0,2 & 2,2 & 1,6 & 1,8 & 1,1 \\
\hline \multirow{2}{*}{ POTASIO (mEq/l) } & $4,6 \pm$ & $3,8 \pm$ & $4,9 \pm$ & $4,0 \pm$ & $3,2 \pm$ & $3,6 \pm$ & $5,5 \pm$ & $5,0 \pm$ & $3,6 \pm$ & $4,1 \pm$ & $4,4 \pm$ & $4,3 \pm$ & $4,9 \pm$ & $4,3 \pm$ \\
\hline & 1,0 & 0,6 & 1,0 & 0,7 & 0,8 & 1,2 & 1,4 & 1,0 & 0,8 & 1,2 & 1,2 & 1,3 & 0,6 & 1,2 \\
\hline Peso (gramos) & 8.525 & 9.250 & 22.700 & 26.650 & 15.500 & 17.800 & 16.100 & 17.000 & 31.250 & 35.000 & 37.500 & 42.400 & 29.600 & 29.800 \\
\hline
\end{tabular}

* Paciente 2 recibió D.P.1. por un periodo inferior a 2 meses.

I = Ingreso al Programa

$\mathrm{C}=$ Control después de 3 meses de diálisis peritoneal intermitente 
nitrógeno ureico, creatinina, calcemia, fosfemia, electrolitos plasmáticos y proteinemia.

En dos pacientes fue necesario reinstalar el catéter en 3 oportunidades por obstrucción. Una de ellas tenía fibrosis quística del higado y hepatomegalia, que facilitaría esta .compljcación ( 2 reinstalaciones).

Una paciente tuvo, pocos días después de instalado el catéter y también al cabo de 2 y medio meses, estando aún en diálisis, absceso de pared abdominal que mejoró rápidamente con e] retiro del catéter. Se presentaron 3 episodios de peritonitis en los 268 procedimientos efectuados, no lográndose aislar el agente causal.

\section{COMENT ARIO}

Actualmente existe en los países desarrollados amplia experiencia, en adultos y en niños, con técnicas de manejo de la insuficiencia renal crónjca terminal. Como etapa previa al transpiante renal se ha usado la DPCA y la DPI, demostrándose ambas muy útiles. Estudios comparativos de ambas técnicas ${ }^{16}$ muestran buen control de la hipertensión arterial, crecimiento lineal fue similar; sólo hubo diferencias en la frecuencia de peritonitis, que fue mayor en DPCA.

La DPI ${ }^{3}, 8,9,13$. La DPI es una eficiente ayuda para mantener a los niños con insuficiencia renal crónica en estado clínico y metabólico satisfactorios, lo que es, fundamental para el trasplante renal. La técnica es simple, relativamente barata en comparación con la DPCA y la hemodiálisis y además segura, ya que en el uso de catéteres de Silastic $y$ adaptadores de Titanio se logró eliminar los riesgos de ruptura intestinal y disminuir la incidencia de peritonitis ${ }^{10}, 21$. En relación con la infección del peritoneo, hemos modificado los criterios de diagnóstico, pues la turbidez del líquido de diálisis y el aumento de la celularidad no son indicadores por sí solos de infección, a menos que se acompañen de dolor abdominal, fiebre, $o$ ambos. En 268 procedimientos de diálisis hicimos el diagnóstico y tratamos sólo tres episodios en otros tantos niños. Llama la atención que nunca hemos logrado obtener gérmenes de los cultivos del liquido peritoneal; la explicación más probable es que inicjalmente los cultivos se realizaron con 3 a $5 \mathrm{ml}$ de líquido y fueron cultivados en agar sangre-chocolate. Actualmente tomamos $100 \mathrm{ml}$ de muestra que son incubados en caldo antes de ser cultivados en agar, con lo que esperamos obtener información más precisa.

E] pequeño tamaño de la muestra no permitió analizar la evolución pondoestatural, pero pudimos establecer que se produjo crecimiento acelerado en todos los niños en que había concordancia entre la edad ósea y la cronológica, no sucediendo lo mismo cuando la edad osea estaba atrasada de manera significativa. Estos resultados son concordantes con lo sucedido en los niños que han recibido trasplantes renales ${ }^{27}$.

Con el empleo de DPI el estado ácido-básico sufre una rápida y sostenida mejoría, en tanto que los electroljtos plasmáticos son más lentos en normalizarse, pero también se mantienen muy estables. Los niveles de calcio y fósforo en sangre mejoran de manera significativa, aunque lenta. En cuanto al nitrógeno ureico, experimenta una disminución de 40 a $50 \%$ durante el primer mes de diálisis, para luego estabilizarse en cifras cerca. nas a $40 \%$ de las previas a la diálisis. Las variaciones de la creatinina sérica son menos significativas (descenso de 20 a $30 \%$ ) manteniéndose constante en los meses siguientes, pero nunca alcanzaron los valores normales en nuestros pacientes, siendo una explicación probable que nuestros nínos son dializados sólo dos veces por semana lo que no permite una depuración completa.

Durante el período de tres meses que, en cada caso, incluye la evaluación que presentamos, no se produjo un aumento significativo de la albuminemia de nuestros pacientes, lo que puede ser explicado, al menos en parke, por las pérdidas de proteinas $y$ amino ácidos provocadas por la misma diálisis ${ }^{24}$, la menor ingestión de proteínas relativa a la anorexia producida por la enfermedad y los trastornos psico-sociales que ella trae sobre el paciente y la familia y las variaciones indjviduales e inducidas por el síndrome urémico en la síntesis y catabolismo proteicos 25.26

En todo caso, si bien se requieren estudios que incluyan mayor número de pacientes, los resultados parciales que hemos obtenido con este método son alentadores pues permite un manejo aparentemente adecuado de los niños con insuficiencia renal crónica a un costo relativamente bajo en comparación con hemodiálissis y DPCA y, al menos teóricamente, facilita la participación de la familia y una mejor adaptación de los enfermos al medio y la enfermedad.

\section{RESLIMEN}

Se presenta la experiencia de la diâlisis peritoneal intermitente en 8 niños insuficientes renales crónicos en su etapa previa al transplante renal, entre 1983 y 1985 en el Hospital Luis Calvo Mackenna. 
La muestra comprende seis mujeres. Ia edad de los pacientes fluctuó entre 2 anfos 1 mes y 12 atios 10 meses con un promedio de 9 años 4 meses. En la diálisis se usaron catéteres de Silastic y soluciones gluco-electrolíticas isotónicas a $37^{\circ} \mathrm{C}$, en volúmenes de 30 a $40 \mathrm{ml} \times \mathrm{kg}$ que fueron mantenidas en la cavidad peritoneal por 45 minutos y removidos en 15 minutos, repitiendo el procedimiento 24 veces cada día 2 veces por semana. Se efectuaron en total 268 días de diálisis con un mínimo de $10 \mathrm{y}$ un máximo de 60 por cada paciente.

Cada 15 días se hizo una evaluación antropométrica, bioquímica $y$ hematológica observando mejoría, aunque no normalización de estos parámetros. Sin embargo los pacientes pudieron realizar una vida normal y liegar al trasplante en excelentes condiciones generales.

De los 8 pacientes, 6 recibieron trasplantes renales donados por parientes y 2 esperan rivones de otros donantes.

En 2 pacientes se obstruyeron los catéteres de diálisis por lo que hubo que removerlos; registramos 3 episodios de peritonitis bacteriana, en los que no se logró aislar el germen causal.

Nuestros resultados parciales parecen buenos y como el sistema es barato y simple en comparación con la hemodiálisis y la diálisis peritoneal continua arribulatoria, creemos que la diálisis peritoneal intermitente es un procedimiento recomendable en países en desarrollo que no cuentan con los medios de financiamiento necesario para aplicar términos que por ahora son más costosas.

\section{REFERENCLAS}

1. Fine R.N. and Gruskin A.B.: End stage renal disease in children W.B. Saunders Co. 1st. Edition. 1984,

2. Mac Donald $H_{\text {: }}$ : Discussion of Trans. Am. Soc. Artif. Int. Organs 10: 419, 1964.

3. Boen S.T., Mulinari A.S., Dillard D.H., Scribner $B . H$. Periodic peritoneal dialysis in the management of chronic uremia. Trans, Am. Soc. Artif. Int. Ongans B: 256, 1962.

4. Losker N.: Chronic peritoneal dialysis. Pa. Med. 74 : 67, 1971.

5. Boen S.T. Kinetics of peritoneal dialysis. A comparison with the artificial Kidney. Medicine (Baltimore) 40: 243, 1961.

6. Tenkhoff H., Maston B., Shilipeter G.: A simplified automatic peritoneal dialysis system. Trans. Am. Soc. Artif. Organs $18: 436,1972$.

7. Bonzel K.E., Dieckman L.. Koch H.: Chronic peritoneal dialysis in children. Pediatric Res 14: 994 , 1980 .
8. Feldman W., Babiah T., Drumond K.N.: Intermittent peritoneal dialysis in the management of chronic renal failure in children. Am. J. Dis. Child. 116: 30, 1969.

9. García O.R., Duclos H.J.: Dialisis peritoneal crónjca intermitente. Una alternativa para la insuficiencia renal crónica terminal, Rev. Med. Chile 112: 139,1984

10. Tenkhoff H.: Peritoneal dialysis A new look. Nephuon 12: 420, 1974 ,

11. Gruskin A.B., Elzouky A.Y., Baluarte H.J.: Petitoneal dialysis kinetics-pediatrics perspective In; $\mathrm{Pe}$ diatrics Nephrology. Vol. VI Strauss J. (Ed.) New York, Plenum Press 1981. Pag. 439.

12. Esperanca M.J., Collins D.L.: Peritoneal dialysis efficiency in relation to body weight. J.. Pediatr. Sutg. 1: 163, 1966.

13. Day R.E., White R.H.R.: Peritoneal dialysis in children, Review of 8 years of experience. Arch. Dis. Child, 52: $56,1977$.

14. Lugo $G$, Ceballos $R$., Brown W.: Acute renal failure in the neonate managed by peritoneal dialy sis. Am. J. Dis. Child, 116: 655, 1969.

15. Been $S . T$. Kinetics of peritoneal dialy sis. Medicine 40: $243,1961$.

16. Baluarte J., Gruskin A., Polinsky M., Prebis J, Perlman Sh., Morgenster B.. Comparison of chronic intermittent and continuous ambulatory peritoneal dialysis in children. Pediatr. Res. 16, 31B-A, 1982.

17. Rife M.: Peritoneal Dialysis. What's ahead? Dial, Traspl. 11. 663, 1982.

18. Brewer $T$., Cakiwell $F_{1}$, Paterson $R$, Flangan $w$ : Indwelling peritoneal dialysis catheter. JAMA 219: 1011,1972

19. Balfe J.W., Irwing M.A., Oreopoutos D.G.: An assesment of CAPD in children. In: Moncrief J.W. and Popovich R.P. (Ed), C.APD Update, Masson Publishing U.S.A. Ine. 1981 Pag. 211.

20. Alexander S.R., Tank E.S.: Surgical aspect of CAPD in infants, chifren and adolescents. J. Lrolog. 127: 501, 1981.

21. Saieh C., Cordero J., Rodriguez E., Puga F., Varelo M., Hernóndez $C$. D Dálisis en Pediatría. Enviado a publicación (Rev. Chil, Pediats.).

22. Martinez L., Alarcón F., Sajeh C., Vaccarezza A., Martinez $F$. Crecimiento después del trasplante renal en relación al potencial de crecimiento. I Congreso Latinoamericano de Nefrología Pediátrica. Viña del Mar, Chile. Octubre, 1984.

23. Soutwest pediatric nephrology study group.: Continuous anbulatory and continuous cycling peritoneal dialysis in chíldren. Kidney Jnt. 27: 558 , 1985.

24. Balfe W., Stefanidis C.J., Steel B.T., Hewitt Y.J.: Continuous ambulatory peritoneal dialysis. Clinical aspects. In: Fine R.N. and Gruskin A.B. End stage renal disease in children. W.B. Saunders Company. Ist. Ed. 1984. Pag. 134.

25. Biumenkrantz M.J., Kople J.D., Guttman R.A.: Method for assesing nutritional status of patients with renal failure. Am. J. Clin. Nutr, 33; 1567. 1980.

26. Bianchi R., Mariani G., Gausseppina M., Carmassi T.: The metabolism of human serum albumin in renal failure on conservative and dialysis therapy. Am, J. Clin. Nutr. 31: 1615, 1978. 